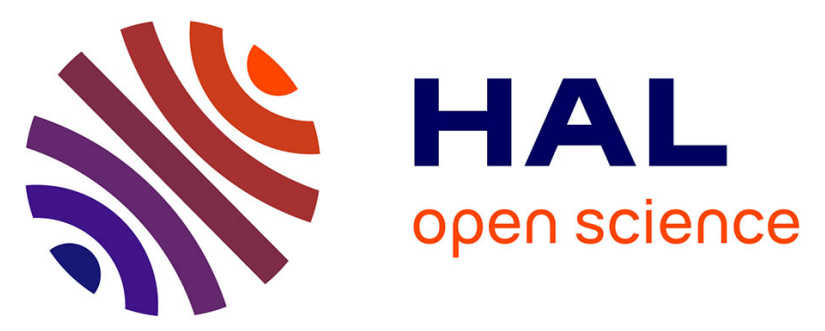

\title{
Effet des bactéries sur la corrosion d'un acier en contexte nucléaire : caractérisation par une approche multi-techniques (microscopie optique, $\mu$ Raman et MEB-EDS)
}

Florence F. Mercier-Bion, Yoanna Leon, Delphine D. Neff, Laurent Urios, Wittebroodt Charles, Margot Flachet, Philippe Dillmann

\section{To cite this version:}

Florence F. Mercier-Bion, Yoanna Leon, Delphine D. Neff, Laurent Urios, Wittebroodt Charles, et al.. Effet des bactéries sur la corrosion d'un acier en contexte nucléaire : caractérisation par une approche multi-techniques (microscopie optique, $\mu$ Raman et MEB-EDS). Matériaux \& Techniques, 2017, 104, pp.511. 10.1051/mattech $/ 2017019$. cea-01634522

HAL Id: cea-01634522

https://hal-cea.archives-ouvertes.fr/cea-01634522

Submitted on 14 Nov 2017

HAL is a multi-disciplinary open access archive for the deposit and dissemination of scientific research documents, whether they are published or not. The documents may come from teaching and research institutions in France or abroad, or from public or private research centers.
L'archive ouverte pluridisciplinaire HAL, est destinée au dépôt et à la diffusion de documents scientifiques de niveau recherche, publiés ou non, émanant des établissements d'enseignement et de recherche français ou étrangers, des laboratoires publics ou privés. 


\title{
Effet des bactéries sur la corrosion d'un acier en contexte nucléaire : caractérisation par une approche multi-techniques (microscopie optique, $\mu$ Raman et MEB-EDS)
}

\author{
Mercier-Bion Florence ${ }^{1, a}$, Leon Yoanna ${ }^{1}$, Neff Delphine ${ }^{1}$, Urios Laurent ${ }^{2}$, \\ Wittebroodt Charles ${ }^{3}$, Flachet Margot $^{4}$ et Dillmann Philippe ${ }^{1}$
}

Reçu le 19 juin 2016, accepté le 24 février 2017

\begin{abstract}
Résumé - Une expérience innovante de mise en corrosion a été réalisée dans ce travail : des coupons d'acier au carbone ont été corrodés pendant neuf mois à $25^{\circ} \mathrm{C}$ au contact d'argilite du Toarcien provenant de Tournemire, d'une solution simulant l'eau porale et en présence ou non d'un cocktail de bactéries représentatives de la biodiversité en milieu argileux profond. Les expériences de corrosion ont été menées en conditions aérées, étape intervenant après la fermeture des dispositifs de stockage des déchets nucléaires et précédant la transition oxique-anoxique. Une approche multi-techniques a été choisie pour caractériser les produits de corrosion et ceux d'origine biologique : microscopie optique, spectroscopie $\mu$ Raman, microscopie électronique à balayage à effet de champ (MEB-FEG) couplée à l'analyse EDS. L'ensemble des techniques a permis de montrer que le système aéré évolue vers un système désaéré lorsque les bactéries sont ajoutées à l'expérience de corrosion.
\end{abstract}

Mots clés : Corrosion / acier / cocktail de bactéries / $\mu$ Raman / MEB-FEG

\section{Introduction}

En France, L'Agence nationale pour la gestion des déchets radioactifs (Andra) propose de stocker en couche géologique profonde les déchets nucléaires de haute et moyenne activité à vie longue. Cette roche hôte consiste en une formation d'argilite du Callovo-Oxfordien située dans l'Est du Bassin Parisien (Bure, Meuse HauteMarne). Dans le concept à l'étude, les déchets sont vitrifiés et le matériau obtenu est placé dans un conteneur en acier inoxydable, formant ainsi le « colis primaire ». Celui-ci est ensuite disposé dans un surconteneur en acier non ou faiblement allié. L'ensemble du colis primaire et du surconteneur forme le « colis de stockage ». Il est ensuite envisagé d'introduire les colis de stockage dans des « alvéoles de stockage » creusées au sein de l'argilite et isolées de celle-ci par un chemisage en acier non allié.

Des conditions anoxiques stables doivent prévaloir dans un tel milieu géologique profond. Ces conditions auraient lieu après une période aérée qui durerait jusqu'à consommation complète de l'oxygène introduit dans le stockage avant la fermeture des alvéoles de stockage et estimée à quelques années. À la suite à cette période aérée, viendrait une étape transitoire aérée-désaérée prévue pour une dizaine d'années $[1,2]$.

Comme une diversité bactérienne a été rapportée en milieu argileux profond (bactéries endogènes) $[3,4]$ et que des microorganismes sont introduits lors des activités humaines de conception des dispositifs de stockage (bactéries exogènes), le rôle des bactéries dans la corrosion des aciers doit être étudié, si l'on veut avoir une vision complète de leur altération.

Dans ce contexte, l'Institut de Radioprotection et de Sureté Nucléaire (IRSN) mène des recherches au sein de la station expérimentale souterraine de Tournemire située dans l'Aveyron. Ce laboratoire souterrain consiste en un ancien tunnel de chemin de fer traversant une formation d'argilite toarcienne datant de 180 millions d'années. Cette couche géologique est particulièrement intéressante car elle présente des propriétés physico-chimiques proches

\footnotetext{
a Auteur de correspondance : florence.mercier@cea.fr

1 LAPA/NIMBE/IRAMIS Université Paris-Saclay Centre CEA de Saclay, 91191 Gif-sur-Yvette, France

2 EEM/IPREM/Université de Pau et des Pays de l'Adour, 64013 Pau, France

3 IRSN/PRP-DGE/SRTG/LETIS, B.P. 17, 92262 Fontenay-aux-Roses, France

${ }^{4}$ RSN/PRP-DGE/SEDRAN/B4S, B.P. 17, 92262 Fontenay-aux-Roses, France
} 
de celles du Callovo-Oxfordien prévue pour recevoir les déchets nucléaires.

La corrosion de l'acier peu allié utilisé comme une des barrières d'enrobage du colis de déchets nucléaires est un des principaux phénomènes à prendre en compte pour le dimensionnement du stockage des déchets nucléaires. Le rôle des bactéries dans la corrosion des aciers est un phénomène bien connu pouvant conduire à l'augmentation des vitesses de corrosion des aciers. Les bactéries peuvent ainsi conduire à une perte d'étanchéité des matériaux d'enrobage des colis de déchets nucléaires car elles catalysent les réactions de corrosion des aciers $[5,6]$.

En milieu argileux profond, la présence de bactéries doit être prise en considération puisque la corrosion anoxique des containers en acier et la radiolyse peuvent produire du dihydrogène, une source énergétique pour le développement des bactéries hydrogénotrophes comme les bactéries sulfato-réductrices (SRB) et les bactréies ferri-réductrices (IRB) [3,7-9].

$\mathrm{Au}$ sein du laboratoire souterrain de Tournemire, une diversité microbienne a été décrite $[4,10]$. Les microorganismes ont été cultivés à partir d'échantillons variés issus de l'argilite : des bactéries aérobies ont été identifiées mais aussi des SRB [4]. La diversité microbienne à l'interface entre des coupons d'acier et de l'argilite recompactée après dix ans in situ a aussi été étudiée : des SRB, des IRB et des souches capables de se développer à de hautes températures ont été détectées [10]. Ces microorganismes peuvent donc se développer aux interfaces acier/argilite sur de courtes périodes comparées aux milliers d'années prévus pour le stockage des déchets nucléaires.

Principalement les SRB et en moindre mesure, les IRB ou les bactéries ferro-oxydantes (IOB) ont été considérées dans les études de biocorrosion des aciers.

Plusieurs types de corrosion peuvent intervenir avec des bactéries SRB en milieu anoxique en présence de sulfates. Le métabolisme des SRB se fait par la réduction des sulfates en sulfure d'hydrogène avec des électrons qui viennent de la matière organique du milieu ou du dihydrogène (on parle dans ce cas de CMIC ou Chemical Microbially Influenced Corrosion) ou venant directement du fer métal (on parle alors de EMIC ou Electrical Microbially Influenced Corrosion) lorsque les environnements sont pauvres en matière organique ou lorsque certaines souches SRB récemment révélées sont impliquées [11,12].

Concernant l'étude des bactéries métabolisant le fer, Ashassi-Sorkhabi et al. [13] ont étudié l'influence des IOB sur la corrosion des aciers au carbone et ont montré que ces souches accroissent les vitesses de corrosion. D'autres auteurs $[14,15]$ ont rapporté l'effet des IRB dans la corrosion des aciers au carbone. S'agissant des IOB, les auteurs ne proposent pas de mécanismes ni aucune réaction pour expliquer l'accroissement des vitesses de corrosion des aciers au carbone quand les bactéries IOB sont impliquées. Il est par contre rapporté que les IOB aboutissent à des piqûres de corrosion. Concernant les IRB, leur rôle en biocorrosion est encore sous discussion puisque ces bactéries peuvent accélérer ou inhiber la corrosion des aciers. L'augmentation de la corrosion viendrait du fait que les IRB conduisent à la réduction dissimilatrice du $\mathrm{Fe}(\mathrm{III})$ en $\mathrm{Fe}(\mathrm{II})$ (par contact direct bactéries-oxydes, transfert d'électrons par substances organiques exogènes du milieu ou par le biais des pili des bactéries, appelés «nanowires ») des films d'oxydes et d'oxy-hydroxydes formés en surface du métal et de la solubilisation subséquente de ces films. Ainsi, la surface métallique se trouve davantage exposée au milieu corrosif ce qui accélère sa corrosion $[14,15]$. Les bactéries IRB sont capables de créer des zones anaérobies qui peuvent favoriser la croissance de bactéries SRB quand ces dernières sont aussi présentes dans le consortium bactérien. Au contraire, les bactéries IRB peuvent conduire à une inhibition de la corrosion $[14,15]$ quand les exopolysaccharides (EPS) des biofilms formés par certaines souches IRB empêchent la dissolution des produits de corrosion de $\mathrm{Fe}^{2+}$. Dans ce cas, les EPS agissent comme une barrière entre le métal et son environnement [16].

Même si la bibliographie en lien avec la biocorrosion est très riche, la plupart des études concerne l'influence d'une souche bactérienne, soit une souche SRB, soit une souche IRB.

À notre connaissance, l'impact de plusieurs grands groupes métaboliques sur la corrosion d'un acier avec le suivi de l'effet synergique ou inhibiteur des souches bactériennes entre elles, n'a jamais été étudié.

Dans cette étude, des coupons en acier au carbone ont été corrodés au contact d'argilite de Tournemire, en présence ou en l'absence d'un cocktail de bactéries ajouté, en conditions aérées pendant neuf mois. Ces conditions ont été choisies pour simuler la période aérée qui intervient lors de la fermeture des dispositifs de stockage des déchets nucléaires, juste avant la période transitoire oxique-anoxique et la période anoxique définitive. Les bactéries ajoutées ont été choisies pour être représentatives des groupes métaboliques présents en milieu géologique profond $[4,10,15]$ : SRB, IRB, IOB, bactéries sulfo-oxydantes (SOB) et des « souches formant des biofilms $»$ n'ayant elles aucune activité de type SRB, IRB, IOB ou SOB $[17,18]$.

Une approche analytique multi-techniques a été choisie pour étudier la corrosion : la microscopie optique permet de localiser les produits de corrosion et les formes bactériennes en surface de l'acier, la micro-spectroscopie Raman ( $\mu$ Raman) donne la nature cristalline des produits de corrosion, la microscopie électronique à balayage à effet de champ (MEB-FEG) donne la morphologie des structures bactériennes et l'analyse EDS couplée permet la détection de leurs éléments constitutifs.

Dans le domaine de la biocorrosion des aciers et ses différentes applications tant dans le domaine du nucléaire ou de l'industrie pétrolière, l'approche est souvent multidisciplinaire, alliant la caractérisation des produits de corrosion ou/et des structures organiques (bactéries, biofilms, EPS) $[19,20]$ souvent en complément de mesures électrochimiques et de la détermination des vitesses de corrosion des aciers [13,15, 21-25]. Cependant, à notre connaissance, le couplage de différentes techniques analytiques pour avoir à la fois l'information sur la nature des 


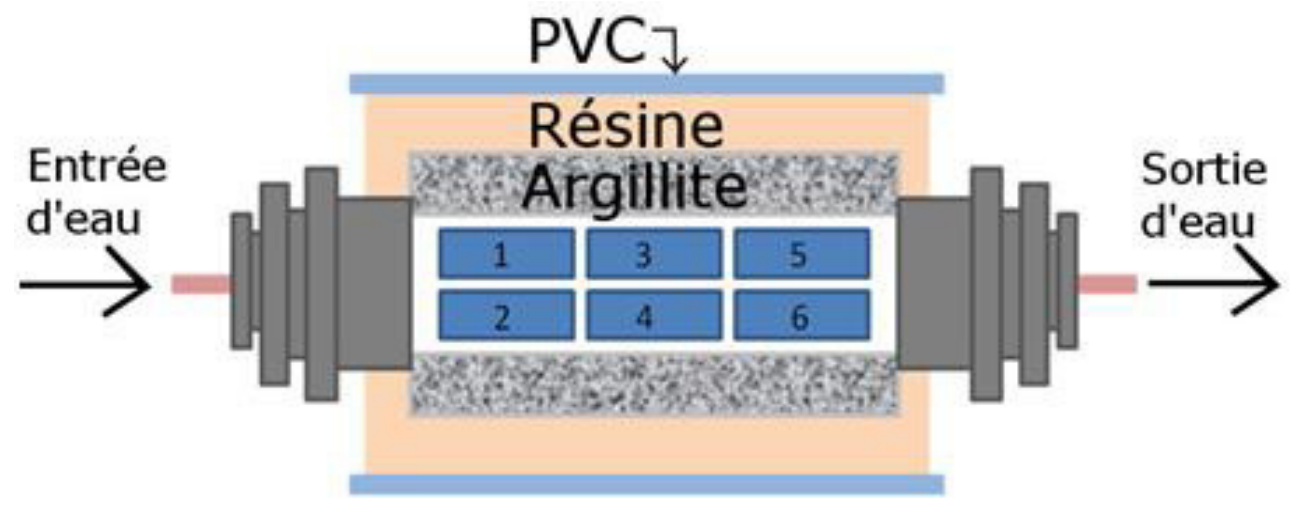

Coupons d'acier

Fig. 1. Représentation schématique de la cellule de corrosion avec les six coupons d'acier à l'intérieur.
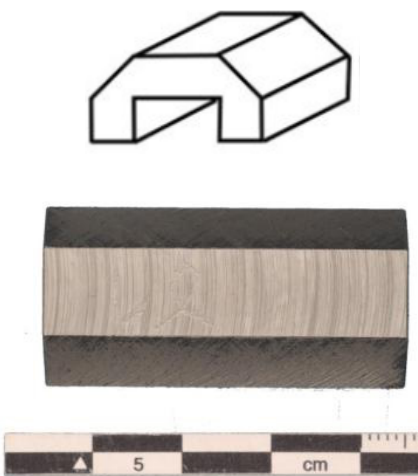

Fig. 2. Schématisation et photographie d'un coupon d'acier.

produits de corrosion mais aussi sur celle des composés organiques à une échelle microscopique, n'a été que très peu exploité. Des techniques d'imagerie comme le MEB ont été utilisées, en particulier pour mettre en évidence les biofilms en surface des aciers [20] ou la morphologie des produits de corrosion [13].

L'approche multi-techniques alliant à la fois la détermination des produits de corrosion avec celle des structures organiques a aussi été exploitée dans les travaux d'AlAbbas et al. [22-24] avec une complémentarité des mesures électrochimiques, de la microscopie électronique à balayage et de la diffraction des rayons X pour l'étude des aciers des oléoducs corrodés par des SRB ou des IRB. Cependant, dans cette étude, aucune information n'est donnée sur la morphologie et la nature des produits à l'échelle microscopique puisque les mesures DRX sont macroscopiques.

Dans notre article sont présentés les résultats de la corrosion aqueuse aérée des coupons d'acier au carbone au contact d'argilite avec : (1) les données concernant leur corrosion abiotique et (2) celles associées à leur corrosion biotique, en mettant l'accent sur la caractérisation à l'échelle microscopique à la fois des produits de corrosion mais aussi des morphologies bactériennes.

\section{Matériels et méthodes}

\subsection{Expériences de corrosion}

Des cellules d'incubation (Fig. 1) contenant des coupons d'acier au carbone sont mises en place pendant neuf mois à $25^{\circ} \mathrm{C}$ en conditions aérées en l'absence ou en présence d'un cocktail de bactéries.

Les cellules de corrosion consistent en un bloc cylindrique d'argilite de Tournemire, forée en son centre sur toute sa longueur afin de placer les six coupons d'acier au carbone. Les coupons d'acier sont des demi-octogones creux (Fig. 2). Ils ont été dégraissés à l'acétone avant d'être utilisés pour les expériences de corrosion. Chaque bloc d'argilite est entouré de résine et de PVC (Fig. 1). $\mathrm{Au}$ sein des cellules de corrosion, circule, sous un régime hydraulique de percolation lente (environ $15 \mathrm{~mL}$ par semaine), de l'eau de formation reconstituée, représentative de l'eau présente sur le site de Tournemire. La préparation d'un litre de la solution synthétique représentative de l'eau du site de Tournemire requiert les quantités suivantes de sels : 0,7902 $\mathrm{g}$ de $\left(\mathrm{NH}_{4}\right)_{2} \mathrm{SO}_{4}, 0,6052 \mathrm{~g}$ de $\mathrm{NaHCO}_{3}, 0,0817 \mathrm{~g}$ de $\mathrm{KH}_{2} \mathrm{PO}_{4}, 2,3121 \mathrm{~g}$ de $\mathrm{Na}_{2} \mathrm{SO}_{4}$, $0,1082 \mathrm{~g}$ de $\mathrm{NaCl}, 0,3212 \mathrm{~g}$ de $\mathrm{MgCl}_{2}$ et $0,5049 \mathrm{~g}$ de $\mathrm{CaCl}_{2} \cdot 2 \mathrm{H}_{2} \mathrm{O}$. La composition de l'eau utilisée ici a été choisie d'après la thèse de Chautard [26] qui approche au maximum la composition de l'eau porale en équilibre avec la roche argileuse de Tournemire [27, 28]. À l'eau de Tournemire reconstituée, on ajoute une source de carbone (acétate) pour fournir l'énergie aux bactéries. Le pH est autour de 8 et aucune variation notable du $\mathrm{pH}$ n'a été relevée entre les effluents d'entrée et ceux de sortie de cellule de corrosion.

Pour les cellules de corrosion biotique, a été ajouté un cocktail de bactéries représentatif des groupes métaboliques rapportés en milieu argileux profond [3, 4 , 10] avec une concentration de $1,25 \times 10^{5}$ cellules.mL ${ }^{-1}$ pour chaque souche bactérienne. Le cocktail comprend cinq souches bactériennes : Geobacter chapellei (IRB), Desulfotomaculum thermoacetoxidans (SRB), 
Tableau 1. Métabolisme et conditions de croissance vis-à-vis de l'oxygène des souches bactériennes de l'inoculum ajouté dans les expériences de corrosion biotiques.

\begin{tabular}{|c|c|c|}
\hline Souche bactérienne & Métabolisme & $\begin{array}{c}\text { Conditions de croissanc } \\
\text { (oxique ou anoxique) }\end{array}$ \\
\hline Desulfotomaculum thermoacetoxidans & SRB & oxique \\
\hline Thiobacillus denitrificans & SOB, IOB & oxique, anoxique \\
\hline Geobacter chapellei & IRB & anoxique \\
\hline Virgibacillus proomii & $\begin{array}{l}\text { Souche formant des biofilms } \\
\text { (sans activité SRB, IRB, IOB, SOB) }\end{array}$ & oxique, anoxique \\
\hline Geobacillus subterraneus & $\begin{array}{c}\text { Souche formant des biofilms } \\
\text { (sans activité SRB, IRB, IOB, SOB) }\end{array}$ & oxique, anoxique \\
\hline
\end{tabular}

Thiobacillus denitrificans (SOB and IOB) et des souches formant des biofilms, Geobacillus subterraneus and Virgibacillus promii, n'ayant aucune activité de type SRB, IRB, IOB ou SOB $[17,18]$. Les souches sont aérobies, anaérobies ou anaérobies facultatives, c'est-à-dire qu'elles s'adaptent à un milieu oxique ou anoxique (Tab. 1). La corrosion se fait en milieu aéré par un bullage d'air.

À l'issue des expériences de corrosion de neuf mois, a lieu le démantèlement qui consiste à extraire les six coupons de la cellule de corrosion. Une fois extraits, les coupons sont laissés à sécher sous sorbonne puis stockés dans un dessicateur préalablement à leur caractérisation en termes de produits de corrosion et de composés d'origine biologique, par les différentes techniques d'analyse complémentaires (microscopie optique, $\mu$ Raman, microscopie électronique à balayage avec analyse EDS).

\subsection{Techniques analytiques}

Les couches de produits de corrosion sont caractérisées par microscopie optique (MO), par micro-spectroscopie Raman ( $\mu$ Raman) et par microscopie électronique à balayage en mode électrons secondaires couplée à de la spectroscopie dispersive en énergie (MEB-EDS).

\subsubsection{Micro-spectroscopie Raman ( $\mu$ Raman)}

Les expériences ont été réalisées sur un spectromètre Invia Reflex ${ }^{\circledR}$ en utilisant une longueur d'onde de $532 \mathrm{~nm}$. La puissance laser est de 0,1 $\mathrm{mW}$ pour éviter la dégradation des produits de corrosion. Le diamètre du faisceau et la profondeur d'analyse sont de $1 \mu \mathrm{m}$. La calibration du spectromètre est obtenue à partir de l'analyse d'un cristal de silicium dont la bande Raman principale est à $520,5 \mathrm{~cm}^{-1}$. L'acquisition et le traitement des spectres sont réalisées avec le logiciel Wire $3,4^{\circledR}$.

Le Tableau 2 donne les positions des bandes Raman pour des produits de corrosion formés en milieux aéré et désaéré.

Des mesures en point fixe ou en cartographies $\mu$ Raman ont été réalisées dans notre étude. Pour les pointés, le temps d'acquisition est de $120 \mathrm{~s}$.

Pour les cartographies en mode point par point, le pas est de $1 \mu \mathrm{m}$ et le temps d'acquisition par point est de 120 s. Pour obtenir la répartition de chaque phase dans la zone cartographiée, l'option « Signal to baseline » est choisie dans le logiciel. Pour chaque produit de corrosion, sa distribution dans la zone cartographiée correspond donc à la représentation de l'aire nette de son pic principal. Ensuite, à partir de la distribution des phases de la cartographie, on vérifie que le spectre de chaque pixel correspond bien à la phase mise en évidence sur la cartographie.

\subsubsection{Microscope électronique à balayage à effet de champ (MEB-FEG) et analyse EDS}

Dans le cadre de cette étude, les images en électrons secondaires et les analyses EDS ont été réalisées avec un microscope électronique à balayage à effet de champ JEOL SEM 7001F couplé à un système de Microanalyse EDS-X (SAMx) équipé d'un détecteur SDD (Silicon Drift Detector). Les images en électrons secondaires et les analyses EDS ont été effectuées avec une tension d'accélération des électrons de $5 \mathrm{kV}$ et un courant faisceau de $8 \mathrm{nA}$. Pour les analyses EDS, le temps d'acquisition est de $120 \mathrm{~s}$. Cette tension d'accélération permet de détecter les éléments constitutifs des bactéries ( $\mathrm{C}, \mathrm{N}$, $\mathrm{O}, \mathrm{P})$ et des produits de corrosion $(\mathrm{O}, \mathrm{Fe})$ avec pour la détection de Fe, l'utilisation de la raie $\mathrm{L}$ à $0,8 \mathrm{keV}$.

Les coupons ont été analysés par MEB-FEG sans préparation préalable, pour permettre la caractérisation aisée des produits de corrosion par les autres techniques qui pourrait s'avérer délicate avec les méthodes de préparation classique des bactéries [22-24,34,35].

\section{Résultats}

Les résultats présentés ci-après concernent, en premier lieu, la corrosion des coupons en l'absence de bactéries ajoutées et ensuite la corrosion en présence de bactéries ajoutées.

\subsection{Corrosion en conditions abiotiques}

La surface du coupon n'est pas totalement corrodée comme ceci est observé sur la Figure 3.

La Figure 4 présente une micrographie optique de la morphologie des produits de corrosion présents en surface de ce coupon. 
Tableau 2. Positions en $\mathrm{cm}^{-1}$ des bandes Raman $\left(150-1100 \mathrm{~cm}^{-1}\right)$ pour des produits de corrosion formés en conditions aérées ou désaérées. La bande principale est soulignée.

\begin{tabular}{|c|c|c|c|c|c|c|c|}
\hline Goethite $\alpha-\mathrm{Fe}^{\mathrm{III}} \mathrm{OOH}$ & 244 & 299 & $\underline{385}$ & 480 & 548 & 681 & {$[29-32]$} \\
\hline Lépidocrocite $\gamma-\mathrm{Fe}^{\mathrm{III}} \mathrm{OOH}$ & $\underline{250}$ & 348 & 379 & 528 & 650 & & {$[29-32]$} \\
\hline Ferrihydrite $\mathrm{Fe}_{2}^{\mathrm{III}} \mathrm{O}_{3} \cdot \mathrm{nH}_{2} \mathrm{O}$ & 370 & 510 & $\underline{710}$ & & & & {$[29-32]$} \\
\hline Magnétite $\mathrm{Fe}^{\mathrm{II}} \mathrm{Fe}_{2}^{\mathrm{III}} \mathrm{O}_{4}$ & 310 & 440 & $\underline{670}$ & & & & {$[29-33]$} \\
\hline Maghémite $\gamma-\mathrm{Fe}_{2}^{\mathrm{III}} \mathrm{O}_{3}$ & 350 & 512 & $\underline{665}$ & 730 & & & [29-33] \\
\hline Sidérite $\mathrm{Fe}^{\mathrm{II}} \mathrm{CO}_{3}$ & 184 & 287 & 731 & 1090 & & & [29-33] \\
\hline Chukanovite $\mathrm{Fe}_{2}^{\mathrm{II}}(\mathrm{OH})_{2} \mathrm{CO}_{3}$ & 128 & 193 & 383 & 513 & 727 & $\underline{1071}$ & {$[31,33]$} \\
\hline
\end{tabular}

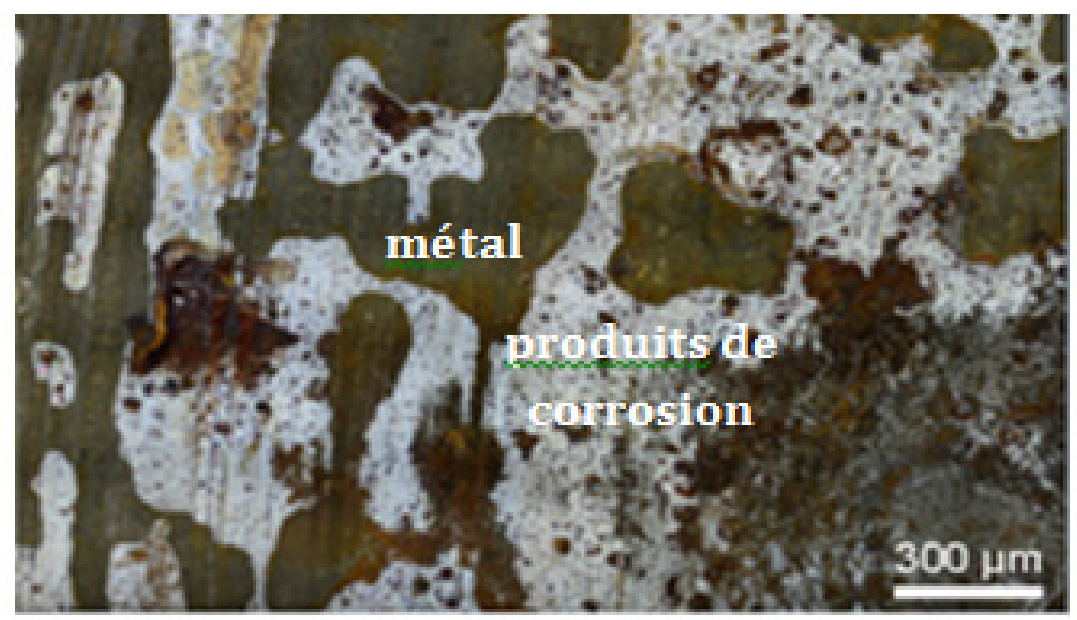

Fig. 3. Micrographie optique de la surface du coupon corrodé en conditions abiotiques.

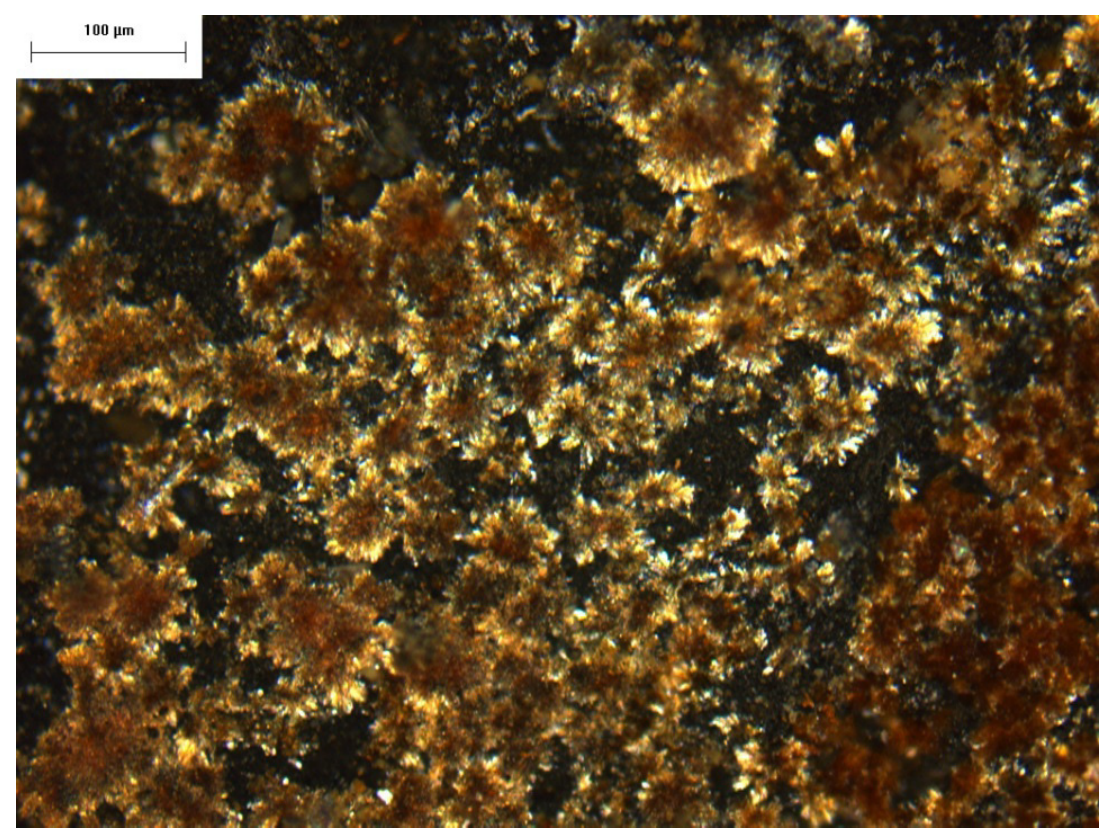

Fig. 4. Micrographie optique de la morphologie des produits de corrosion en surface du coupon corrodé en conditions abiotiques. 
Ces morphologies font penser à celles rapportées dans la bibliographie, dénommées «flowery structures » et attribuées à des oxy-hydroxydes de fer(III) [36-39].

La caractérisation microscopique de ces produits de corrosion par $\mu$ Raman met en évidence deux types de spectres (Fig. 5), par comparaison avec la littérature [30] : un spectre de goethite $\alpha$-FeOOH avec sa bande principale à $391 \mathrm{~cm}^{-1}$ (a) et de lépidocrocite $\gamma$-FeOOH avec sa bande principale à $250 \mathrm{~cm}^{-1}(\mathrm{~b})$.

L'ensemble des techniques complémentaires met en évidence que le coupon corrodé sans bactéries ajoutées présente, comme seuls produits de corrosion, des oxyhydroxydes de fer(III) typiques d'une corrosion en milieu aéré.

\subsection{Corrosion en conditions biotiques}

Contrairement au coupon corrodé sans bactéries, la surface du coupon est totalement corrodée (Fig. 6).

La Figure 7 présente les micrographies optiques de la surface du coupon et les deux types de morphologies observées : les morphologies de type «flowery structures » (a), identiques à celles mises en évidence en surface du coupon corrodé sans bactéries et des zones plates, fissurées par endroits et constellées de taches sombres (b).

Ces taches sombres en surface des zones plates et fissurées par endroits, sont attribuées à des empreintes de bactéries, bien mises en évidence sur l'image MEB-FEG en électrons secondaires (Fig. 8a). Le spectre EDS typique correspondant à un pointé d'analyse sur une empreinte de bactérie révèle systématiquement la présence d'un pic de carbone intense mais aussi des signaux d'azote et de phosphore, confirmant la présence de composés d'origine biologique en surface du coupon (Fig. 8b). Ces empreintes observées en surface du coupon ressemblent parfaitement à celles mises en évidence par MEB en surface d'acier biocorrodé et attribuées à des bactéries [24,40]. Ainsi, les analyses EDS à partir de pointés dans les empreintes de bactéries permettent de mettre en évidence les éléments caractéristiques des bactéries à savoir $\mathrm{C}, \mathrm{N}, \mathrm{P}$ tandis que les analyses EDS à partir de pointés en dehors des empreintes de bactéries, dans les zones de type «flowery structures $»$ donnent un spectre EDS avec uniquement les éléments $\mathrm{O}$ et $\mathrm{Fe}$ signant des produits de corrosion.

La Figure 9 représente une micrographie optique d'une zone plate couverte de formes bactériennes en surface du coupon et les cartographies $\mu$ Raman des produits de corrosion présents dans cette zone (b). La distribution des produits de corrosion dans une telle zone montre la présence de phases réduites du fer à proximité des bactéries : magnétite $\mathrm{Fe}^{\mathrm{II}} \mathrm{Fe}_{2}^{\mathrm{III}} \mathrm{O}_{4}$ mais aussi la présence localisée de carbonates de fer(II), comme la sidérite $\mathrm{FeCO}_{3}$ (c).

Ainsi, à l'échelle macroscopique mais aussi microscopique, les conditions biotiques de corrosion conduisent à la formation de phases réduites du fer, comme la magnétite $\mathrm{Fe}_{3} \mathrm{O}_{4}$ et la sidérite $\mathrm{FeCO}_{3}$ et la présence de formes bactériennes au côté de ces composés.

\section{Discussion}

La combinaison des différentes techniques (de macroscopique à microscopique) ont permis de recueillir les informations reportées dans le Tableau 3.

En conditions abiotiques, la surface du coupon n'est pas corrodée totalement et les produits de corrosion sont uniquement des oxy-hydroxydes de fer(III) comme la lépidocrocite et la goethite.

$\mathrm{Au}$ contraire, en conditions biotiques, la surface du coupon est totalement corrodée et la nature des produits de corrosion change : la présence des phases de fer(III) a considérablement diminué au profit de phases réduites comme la magnétite surtout et très localement la présence de phases de fer(II) telle que la sidérite. Les morphologies bactériennes se retrouvent systématiquement au côté des phases réduites. Aucune signature de l'action des bactéries SRB n'a été mise en évidence : en effet aucune phase de sulfure de fer n'a été détectée en surface du coupon. Dans le même sens, aucune évolution significative de la teneur en sulfate entre les effluents d'entrée et de sortie de la cellule de corrosion n'a été observée. Concernant l'action de ce type de bactéries, des travaux mentionnent qu'elles peuvent se développer en milieu aéré en co-culture avec des espèces bactériennes aérobies, avec une consommation rapide d'oxygène dissous par les espèces aérobies [41]. L'action des SRB aurait donc pu se produire puisque le consortium bactérien contient des souches aérobies.

Les conditions biotiques de corrosion aboutissent donc à la formation de phases réduites qui ne se forment pas ou peu (cas de la magnétite) en corrosion aérée [42].

L'ajout de bactéries à l'expérience de corrosion de l'acier en conditions aérées conduit donc à la création de zones localement désaérées dont l'origine pourrait être : (1) l'action de bactéries aérobies venant des conditions non stériles de conception des cellules de corrosion ou/et de l'inoculum; (2) la présence en surface du coupon de morphologies bactériennes comme des biofilms ou EPS, limitant l'accès de l'oxygène comme déjà rapporté dans la bibliographie [43].

Ces zones localement désaérées induiraient un abaissement du potentiel rédox et pouvant ainsi conduire à la formation de phases réduites comme la magnétite $\mathrm{Fe}_{3} \mathrm{O}_{4}$ et des phases de fer(II) comme la sidérite $\mathrm{FeCO}_{3}$, sans l'impact de bactéries ( processus de réduction inorganique »), comme mis en évidence par le diagramme potentiel-pH du système $\mathrm{Fe}-\mathrm{CO}_{2}-\mathrm{H}_{2} \mathrm{O}$ [44].

Les bactéries IRB strictement anaérobies et présentes dans l'inoculum peuvent aussi réduire les oxydes et oxy-hydroxydes de fer(III) (« processus de réduction bactérien $»)$. Ces bactéries sont en effet connues pour réduire les composés du fer(III). Elles utilisent les ions ferriques comme accepteur d'électrons pour le transformer en ions ferreux en conditions anoxiques.

Les études de Herrera et Videla [14] présentent un état de l'art sur l'action des IRB dans la corrosion du fer : ces bactéries ont une action sur la corrosion puisqu'elles sont capables de réduire les films passifs ferriques en surface des aciers au carbone. Généralement, 
M.-B. Florence et al. : Matériaux \& Techniques 104, 511 (2016)

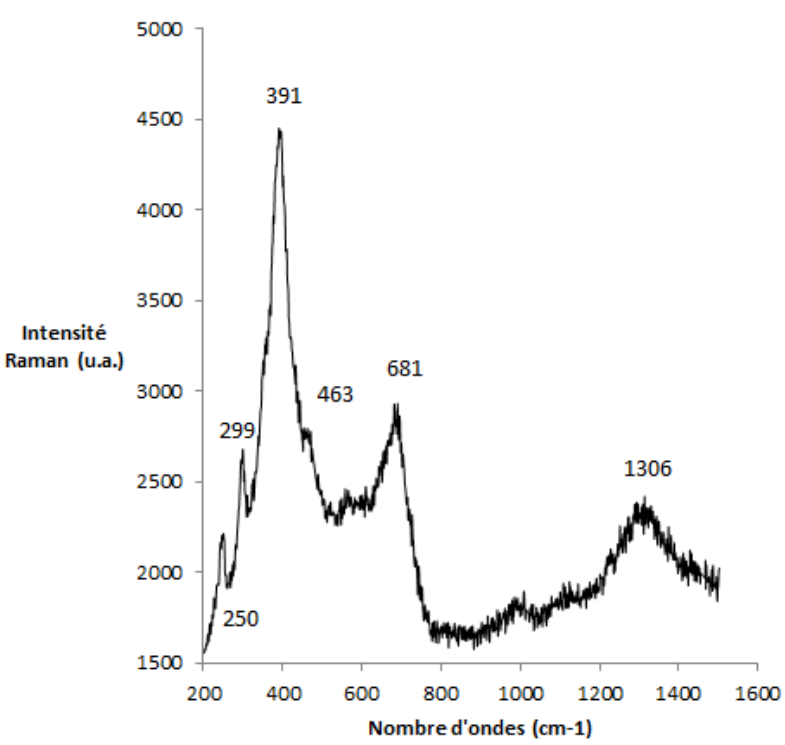

(a)

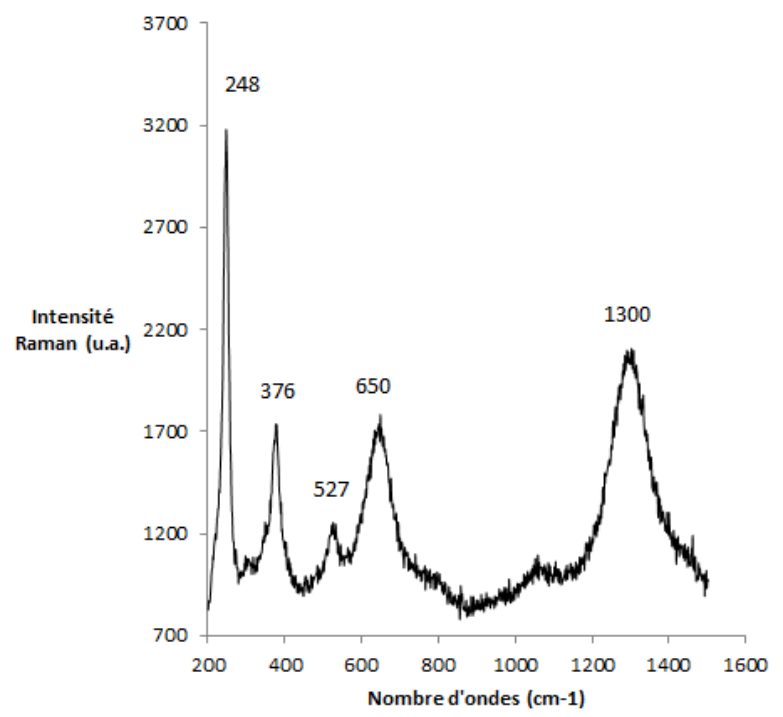

(b)

Fig. 5. Spectres $\mu$ Raman représentatifs des produits de corrosion à la surface du coupon corrodé en conditions abiotiques : goethite $\alpha-\mathrm{Fe}^{\mathrm{III}} \mathrm{OOH}$ (a) et lépidocrocite $\gamma-\mathrm{Fe}^{\mathrm{III}} \mathrm{OOH}(\mathrm{b})$.

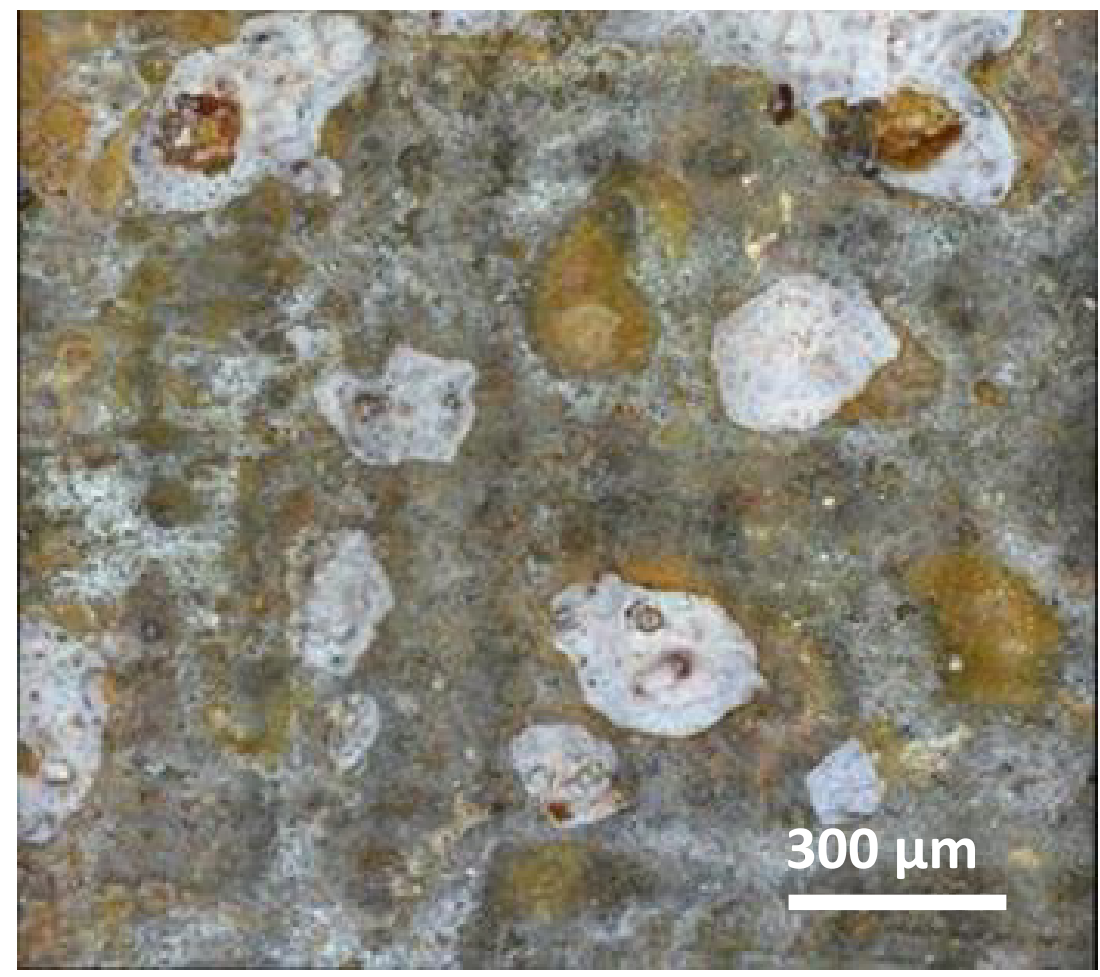

Fig. 6. Micrographie optique de la surface du coupon corrodé en conditions biotiques.

Tableau 3. Résumé des différents résultats obtenus par les différentes techniques en surface des coupons corrodés en conditions abiotiques ou biotiques.

\begin{tabular}{|c|c|c|}
\hline & Conditions abiotiques & Conditions biotiques \\
\hline Produits de corrosion & $\begin{array}{l}\text { Oxy-hydroxydes goethite }\left(\alpha \text {-Fe } \mathrm{Fe}^{\mathrm{III}} \mathrm{OOH}\right) \\
\text { et lépidocrocite }\left(\gamma-\mathrm{Fe}^{\mathrm{III}} \mathrm{OOH}\right)\end{array}$ & $\begin{array}{l}\text { - Diminution des oxy-hydroxydes de fer(III) } \\
\text { - Apparition de plaques fissurées de magnétite }\left(\mathrm{Fe}_{3} \mathrm{O}_{4}\right) \\
\text { avec localement des phases de fer(II) } \\
\text { - Absence de sulfures de fer }\end{array}$ \\
\hline Morphologies bactériennes & $\overline{\mathrm{X}}$ & Formes bactériennes à proximité des phases réduites \\
\hline
\end{tabular}




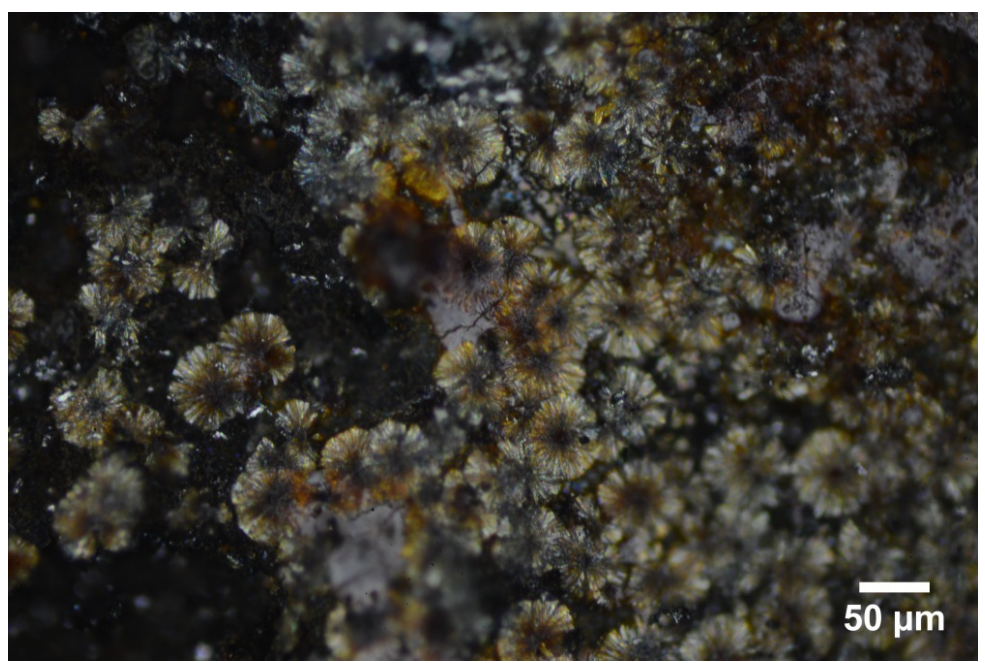

(a)

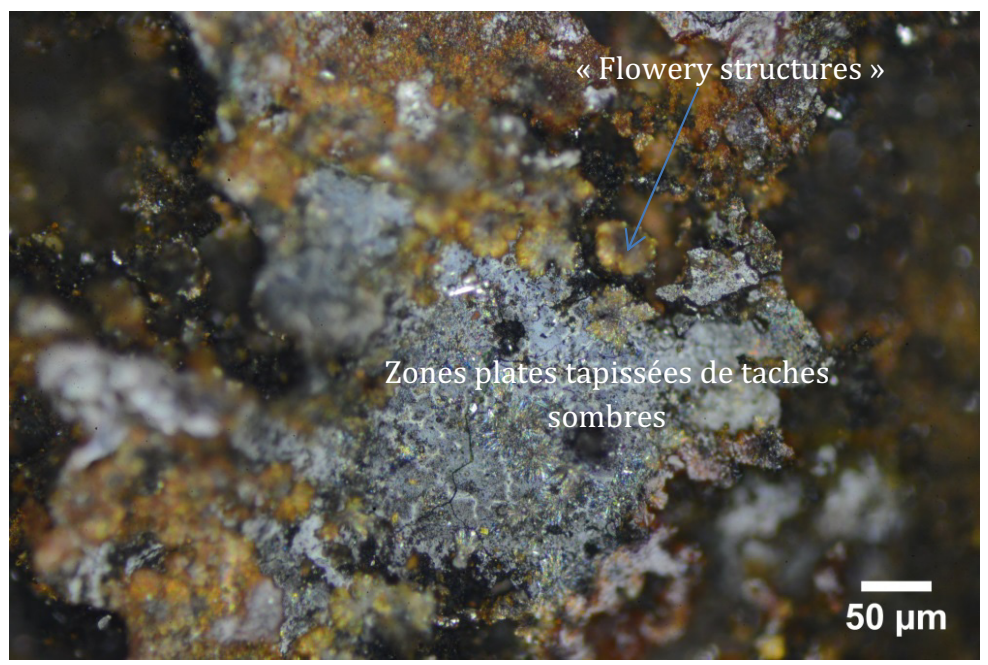

(b)

Fig. 7. Micrographies optiques de la surface du coupon corrodé en conditions biotiques montrant les deux types de morphologie des produits de corrosion : type «flowery structures » (a) et zones plates fissurées et tapissées de taches sombres (b).

la magnétite est la phase secondaire majoritairement obtenue lors de l'activité des IRB, à partir des phases de fer(III) comme la lépidocrocite, la ferrihydrite et la goethite : les études de Zegeye et al. [45] reportent que les souches IRB, Shewanella putrefaciens, anaérobies facultatives, transforment la lépidocrocite $(\gamma-\mathrm{FeOOH})$ en phases secondaires de fer(II)/fer(III) telles que la magnétite et la rouille verte carbonatée.

La réduction microbienne par les IRB des oxydes et oxy-hydroxydes de fer(III) peut conduire à la production de fer(II) et à la formation subséquente de composés de fer(II) incluant la magnétite, la sidérite, la chukanovite et les rouilles vertes $[46,47]$.

$\mathrm{Au} \mathrm{vu}$ de l'ensemble de nos résultats, les deux processus de réduction, « inorganique » ou « bactérien », des composés de fer(III) en composés de fer(II), peuvent être envisagés.
Cependant, quel que soit le processus dominant de réduction des composés de fer(III), cette étude met en évidence qu'une surface d'acier corrodée en présence d'une biodiversité présente en milieu argileux profond dans des conditions de corrosion simulant des conditions in situ lors des phases de construction et de mise en place du dispositif de stockage des colis de déchets nucléaires, présente localement des zones désaérées avec formation de produits de corrosion mixtes $\mathrm{Fe}(\mathrm{III}) / \mathrm{Fe}(\mathrm{II})$ ou de $\mathrm{Fe}(\mathrm{II})$.

Cette observation peut avoir des retombées intéressantes dans les différents domaines d'applications où intervient une corrosion aérée en présence de différentes souches bactériennes.

\section{Conclusion}

Sur un système qui peut être utilisé comme modèle de différentes étapes de stockage des déchets nucléaires 


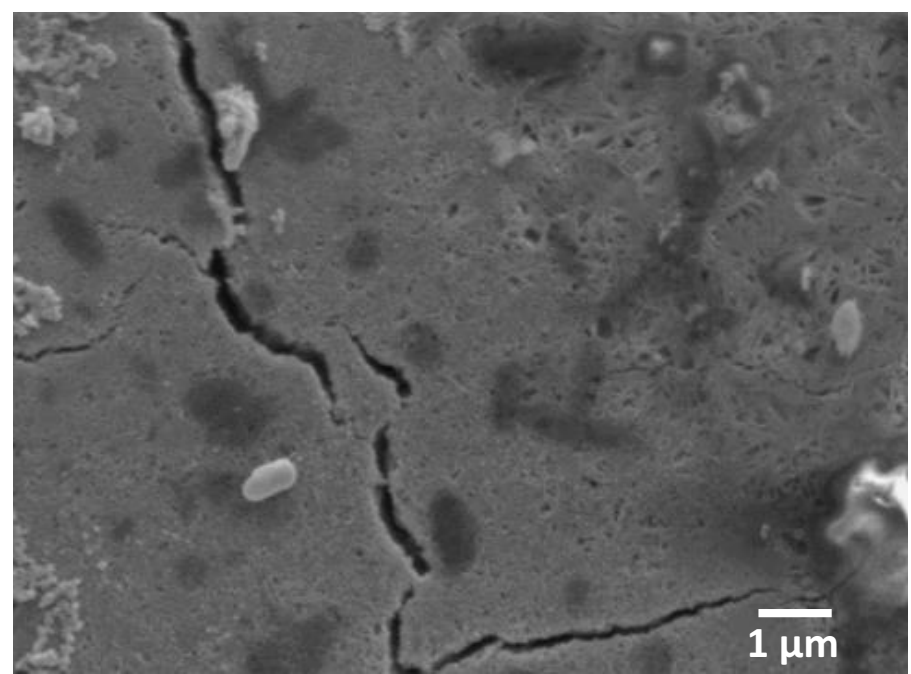

(a)

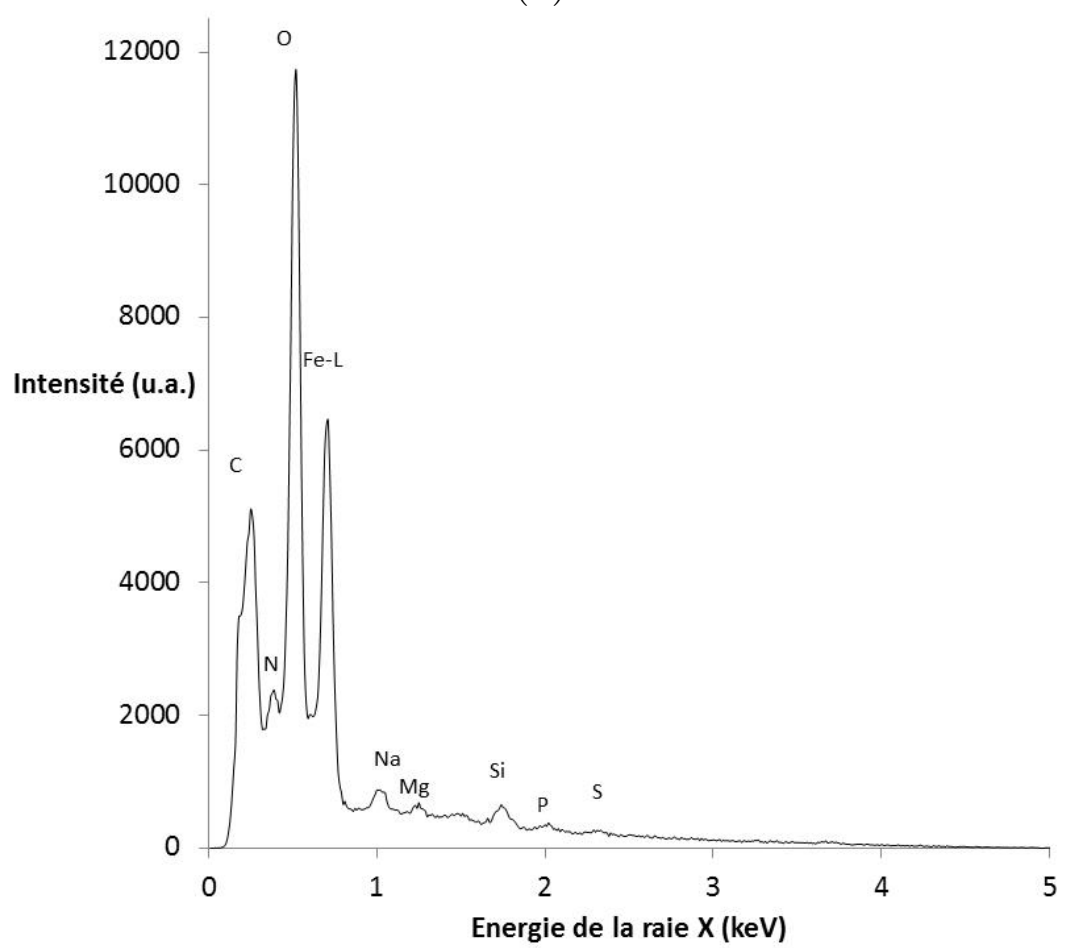

(b)

Fig. 8. (a) Image MEB-FEG en électrons secondaires d'empreintes de bactéries en surface du coupon corrodé en conditions biotiques; (b) Spectre EDS obtenu à partir d'un pointé d'analyse sur une empreinte de bactérie. Tension d'accélération : 5 kV.

en milieu géologique, est étudié la corrosion aérée d'un acier au carbone en l'absence ou en présence de bactéries, représentatives des grands groupes métaboliques présents en milieu argileux profond. L'ensemble des résultats souligne la nécessité de l'utilisation d'une approche multi-techniques pour la caractérisation à l'échelle micrométrique de la surface des coupons d'acier corrodés. Cette approche permet en effet, d'une part, d'avoir les informations sur la localisation et l'identification des produits de corrosion et ceux d'origine bactérienne en surface du coupon biocorrodé, et d'autre part de suivre s'il y a une corrélation entre présence de bactéries sur la surface et nature des produits de corrosion.

Les résultats montrent que, sur le coupon biocorrodé, sont retrouvés localement des produits de corrosion contenant du fer(II) et des morphologies bactériennes. Cette approche a permis de montrer que le coupon d'acier corrodé en milieu aéré en conditions biotiques ne présente pas les mêmes produits de corrosion que celui corrodé en milieu aéré en conditions abiotiques. La contribution des phases de fer(III) diminue au profit de l'apparition de phases de fer(II) comme la magnétite principalement et de 


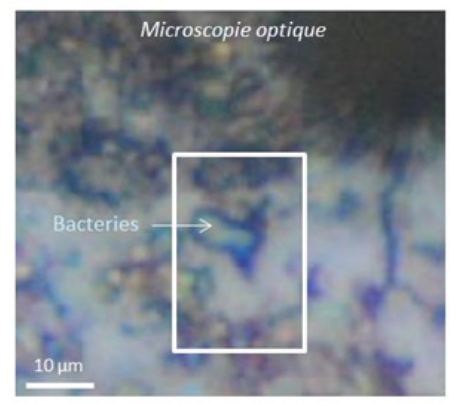

(a)

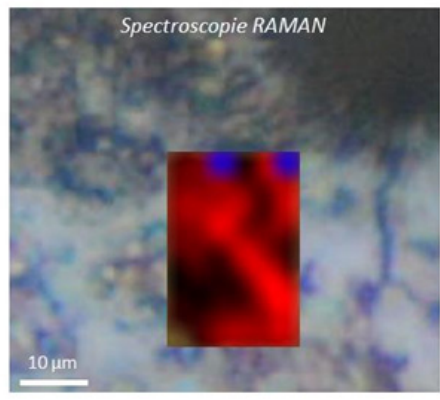

(b)

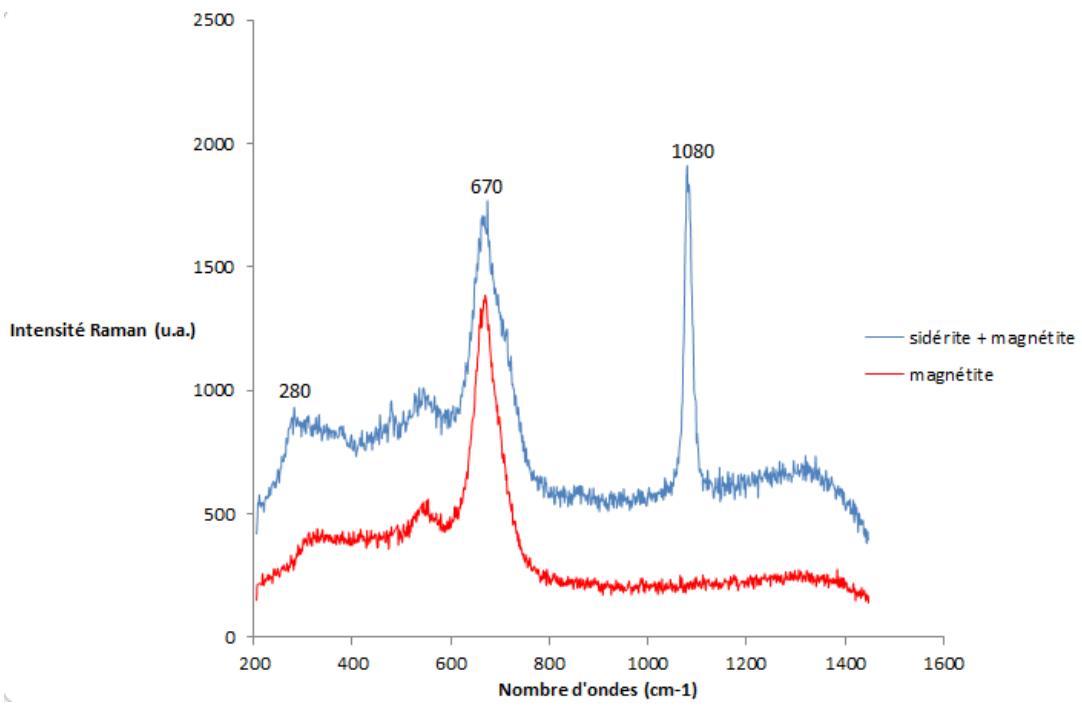

(c)

Fig. 9. Image de microscopie optique (a) et cartographie $\mu$ Raman correspondant à la zone encadrée (b) avec les distributions de la magnétite et de la sidérite. Les spectres $\mu$ Raman correspondant aux deux distributions sont donnés sur la figure (c).

manière localisée des phases de fer(II) comme la sidérite $\mathrm{FeCO}_{3}$, avec la présence systématique de morphologies bactériennes au côté de ces phases.

La suite de cette étude portera sur l'étude de la diversité bactérienne en surface des coupons corrodés en conditions biotiques, pour affiner le mécanisme de formation des phases réduites.

\section{Références}

[1] ANDRA, Evaluation de la faisabilité du stockage géologique en formation argileuse, Dossier 2005 argile (2005)

[2] F. King, Corrosion of carbon steel under anaerobic conditions in a repository for SF and HLW in Opalinus Clay. Technical report 08-12 (Integrity Corrosion Consulting Ltd), 2008

[3] S. Poulain, Caractérisation microbiologique de l'argile à Opalinus du Mont Terri et de l'argilite du CallovoOxfordien de Meuse/Haute-Marne, Thèse Sciences chimiques, 1, CNAB, Bordeaux, 2006

[4] L. Urios, F. Marsal, D. Pellegrini, M. Magot, Appl. Geochem. 27 (2012) 1442-1450
[5] X. Campaignole, J.L. Crolet, Corrosion 53 (1997) 440447

[6] T. Liu, H. Liu, Y. Hu, L. Zhou, B. Zheng, Mater. Corr. 60 (2009) 218-224

[7] S. Stroes-Gascoyne, J.M. West, FEMS Microbiol. Rev. 20 (1997) 573-594

[8] S. Stroes-Gascoyne, A. Schippers, B. Schwyn, S. Poulain, C. Sergeant, M. Simonoff, C. Le Marrec, S. Altmann, T. Nagaoka, L. Mauclaire, J.McKenzie, S. Daumas, A. Vinsot, C. Beaucaire, J.M. Matray, Geomicrobiol J. 24 (2007) 1-17

[9] L. Mauclaire, J.A. Mckenzie, B. Schwyn, P. Bossart, Phys. Chem. Earth 32 (2007) 232-240

[10] L. Urios, F. Marsal, D. Pellegrini, M. Magot, Geomicrobiol. J. 30 (2013) 442-453

[11] D. Enning, J. Garrelfs, Appl. Environ. Microbiol. 80 (2014) 1226

[12] H. Venzlaff, D. Enning, J. Srinivasan, K.J.J. Mayrhofer, A.W. Hassel, F. Widdel, M. Stratmann, Corros. Sci. 66 (2013) 88-96

[13] H. Ashassi-Sorkhabi, M. Moradi-Haghighi, G. Zarrini, R. Javaherdashti, Biodegradation 23 (2012) 69-79

[14] L.K. Herrera, H.A. Videla, Int. Biodeterioration Biodegradation 63 (2009) 891-895 
[15] M.K. Schütz, R. Moreira, O. Bildstein, J.E. Lartigue, M.L. Schlegel, B. Tribollet, V. Vivier, M. Libert, Bioelectrochemistry 97 (2014) 61-68

[16] Videla et Herrera, Int. Biodeterioration Biodegradation 63 (2009) 896-900

[17] M. Heyndrickx, L. Lebbe, K. Kersters, B. Hoste, R. De Wachter, P. De Vos, G. Forsyth, N.A. Logan, Int. J. System. Bacteriol. 49 (1999) 1083-1090

[18] A. Coorevits, A.E. Dinsdale, Halket, Int. J. Syst. Evol. Microbiol. 62 (2012) 1470-1485

[19] B.W.A. Sherar, L.M. Power, P.G. Keech, S. Mitlin, G. Southam, D.W. Shoesmith, Corros. Sci. 53 (2011) 955960

[20] J.W. Arnold, D.H. Boothe, O. Suzuki, G.W. Bailey, J. Microscopy 216 (2004) 215-221

[21] B.W.A. Sherar, P.G. Keech, J.J. Noël, R.G. Worthingham, D.W. Shoesmith, Corrosion 69 (2013) $67-76$

[22] F.M. AlAbbas, C. Williamson, S.M. Bhola, J.R. Spear, D.L. Olson, B. Mishra, A.E. Kakpovbia, Int. Biodeterioration and Biodegradation 78 (2013a) 34-42

[23] F.M. AlAbbas, S.M. Bhola, J.R. Spear, D.L. Olson, B. Mishra, Eng. Failure Analysis 33 (2013b) 222-235

[24] F.M. AlAbbas, C. Williamson, S.M. Bhola, J.R. Spear, D.L. Olson, B. Mishra, A.E. Kakpovbia, J. Mater. Eng. Performance (2013c)

[25] M.K. Schütz, M.L. Schlegel, M. Libert, O. Bildstein, Environ. Sci. Technol. 49 (2015) 7483-7490

[26] Camille Chautard, Interactions fer/argile en conditions de stockage géologique profond - Impacts d'activités bactériennes et d'hétérogénéités, Thèse Paris Tech, 2013

[27] C. Beaucaire, J.L. Michelot, S. Savoye, J. Cabrera, Appl. Geochemistry 23 (2008) 2182-2197

[28] J. Tremosa, D. Arcos, J.M. Matray, F. Bensenouci, E.C. Gaucher, C. Tournassat, Appl. Geochemistry 27 (2011) 1442-1450

[29] D.L.A. de Faria, S. Venancio Silva, M.T. de Oliveira, J. Raman Spectroscopy 28 (1997) 873-878

[30] D. Neff, L. Bellot-Gurlet, P. Dillmann, S. Reguer, L. Legrand, J. Raman Spectroscopy 37 (2006) 1228-1237

[31] M. Saheb, D. Neff, P. Dillmann, H. Matthiesen, E. Foy, L. Bellot-Gurlet, Mater. Corros. 60 (2009) 99
[32] M. Hanesch, Geophys. J. Int. 177 (2009)941-948

[33] M. Saheb, D. Neff, L. Bellot-Gurlet, P. Dillmann, J. Raman Spectrosc. 42 (2011) 1100-1108

[34] K. Qvortrup, J. Rostgaard, P. Bretlau P, Ann. Otol. Rhinol. Laryngol 104 (1995) 120-126

[35] M. Alhede, K. Qvortrup, R. Liebrechts, N. Høiby, M. Givskov, T. Bjarnsholt, Combination of microscopic techniques reveals a comprehensive visual impression of biofilm structure and composition, FEMS Immunol Med Microbiol, 2012, pp. 1-8

[36] R.B. Herbert Jr, Clays and Clay Minerals 45 (1997) 261273

[37] V. Chawla, P.G. Gurbuxani, G.R. Bhagure, J. Minerals Mater. Characterization Eng. 11 (2012) 479-492

[38] J.G. Castano, C.A. Botero, A.H. Restrepo, E.A. Agudelo, E. Correa, F. Echeverria, Corros. Sci. 52 (2010) 216-223

[39] R.A. Antunes, I. Costa, D.L. Araujo de Faria, Mater. Res. 6 (2003) 403-408

[40] B. Little, P. Wagner, K. Hart, R. Ray, D. Lavoie, K. Nealson, C. Aguilar, Biodegradation 9 (1998) 1-10

[41] P. Sigalevich, M.V. Baev, A. Teske, Y. Cohen, Appl. Environ. Microbiol. 66 (2000) 5013-5018

[42] M. Bouchar, E. Foy, D. Neff, P. Dillmann, Corros. Sci. 76 (2013) 361-372

[43] R. Marchal, Rôle des bactéries sulfurogènes dans la corrosion du fer, Oil \& Gas Science and Technology - Rev. IFP 54 (1999) 649-659

[44] J. Chivot, Thermodynamique des produits de corrosion : fonctions thermodynamiques, diagrammes de solubilité, diagrammes E-pH des systems Fe-H2O, Fe-CO2-H2O, Fe$\mathrm{S}-\mathrm{H} 2 \mathrm{O}, \mathrm{Cr}-\mathrm{H} 2 \mathrm{O}$ et Ni-H2O en fonction de la température, Collection Sciences et Techniques, 2004

[45] A. Zegeye, C. Mustin, F. Jorand, Geobiology 8 (2010) 209-222

[46] E.J. O'Loughlin, C.A. Gorski, M.M. Scherer, M.I. Boyanov, K.M. Kemner, Environ. Sci. Technol. 44 (2010) 4570-4576

[47] J.M. Zachara, R.K. Kukkadapu, J.K. Fredrickson, Y.A. Gorby, S. C. Smith, Geomicrobiol. J. 19 (2002) 179-207 